\title{
An Octave Package to Perform Qualitative Analysis of Nonlinear Systems Immersed in $R^{4}$
}

\author{
Eder ESCOBAR ${ }^{\mathrm{a}}$, Richard ABRAMONTE ${ }^{\mathrm{a}}$ Antenor ALIAGA ${ }^{\mathrm{a}}$ and \\ Flabio GUTIERREZ ${ }^{\mathrm{a}, 1}$ \\ ${ }^{a}$ Science Faculty, Universidad Nacional de Piura, Piura, Peru
}

\begin{abstract}
In this work, the AutonomousSystems4D package is presented, which allows the qualitative analysis of non-linear differential equation systems in four dimensions, as well as drawing the phase surfaces by immersing $R^{4}$ in $R^{3}$. The package is programmed in the computational tool Octave. As a case study applied to the new Lorenz 4D System, sensitivity was found in the initial conditions, Lyapunov exponents, Kaplan Yorke dimension, a stable and unstable critical point, limit cycle, Hopf bifurcation, and hyperattractors. The package could be adapted to perform qualitative analysis and visualize phase surfaces to autonomous systems, e.g. Sprott 4D, Rossler 4D, etc. The package can be applied to problems such as: design, analysis, implementation of electronic circuits; to message encryption.
\end{abstract}

Keywords. Octave, Autonomous System, fourth dimension, qualitative analysis

\section{Introduction}

Several physical phenomena are modeled with systems of differentict the phenomenon, but if they do in a fairly approximate way, the confidence in the results of the model is given by the qualitative analysis of the system..

In this work, a package in Octave 5.1.0 called AutonomousSystems4D is presented, which allows qualitative analysis, as well as visualizing phase surfaces of non-linear systems immersed in a four-dimensional (4D) system. As far as we know, there are no similar works.

Currently, to visualize the phase maps and the limit cycles of the systems that are in $4 \mathrm{D}$, they are projected in three dimensions making zero any of the coordinated axes, which will have four surfaces to analyze (see for example [1-3]). In the developed package, no projections are used, the Velezmoro model is used, which allows visualizing 4D objects in a three-dimensional (3D) system (see Section 2.1), so you will have a single surface, which can be rotated from different angles, this allows a better analysis of what happens in the system of $4 \mathrm{D}$ equations. The package could be adapted to perform qualitative analysis and visualize phase surfaces to autonomous systems, e.g. Lorenz 4D,

\footnotetext{
${ }^{1}$ Corresponding Author: Flabio Gutierrez, Department of Mathematics, Universidad Nacional de Piura, Campus Universitario, Urb. Miraflores s/n, Castilla, Piura, Perú; E-mail: flabio@unp.edu.pe
} 
Sprott 4D, Rossler 4D, etc. In this paper we apply it to perform qualitative analysis and visualization of a Lorenz hyper-caotic system immersed in $R^{4}$

Lorenz found the first strange attractor in the three-dimensional autonomous system of equations. In $[4,5]$ qualitative analyses are carried out for this type of system. Works on the Lorenz system in a four-dimensional space can be found in [6-8], these systems are hyper-caotic systems having two or more positive Lyapunov exponents.

The package can be applied to problems such as: design, analysis, implementation of electronic circuits [2,3,9]; to message encryption [10].

\section{Preliminaries}

Definition 1 . Hopf bifurcation [4].

Is the bifurcation corresponding to the presence $\lambda_{1,2}= \pm i w_{0}$, con $w_{0}>0$.

Definition 2 . Break-even point [5].

The point $X=a$ with $f(a)=0$ is called the critical point of the equation $X^{\prime}=f(X)$.

Theorema 1 Lyapunov [4].

Consider a dynamic system defined by $X^{\prime}=F(X)$, where $X \in R^{n}, F$ is continuous.

Suppose you have an equilibrium $X_{0}, A=F_{X}\left(X_{0}\right)$ (A es $A$ is the Jacobian matrix of $F(X)$ evaluated in equilibrium). Then $X_{0}$ is stable if all the eigenvalues $\lambda_{1}, \lambda_{2}, \ldots, \lambda_{n}$ of A satisfy $\operatorname{Re} \lambda<0$.

Definition 3 . In order for the system of $4 D$ equations to be hypercotic it must satisfy three conditions: [6].

1) A four-dimensional autonomous system is required.

2) Sensitivity in the initial conditions.

3) Two or more positive Lyapunov exponents and the sum of all Lyapunov exponents is less than 0 .

Method for finding two roots in the imaginary axis. Consider the cubic equation.

$$
\lambda^{3}+L_{1}(c) \lambda^{2}+L_{2}(c) \lambda+L_{3}(c)=0
$$

Let $\lambda=i w_{0}$ an imaginary solution, then the cubic equation is equivalent to:

$$
i w_{0}\left(L_{2}(c)-w_{0}^{2}\right)+\left(L_{3}(c)-L_{1}(c) w_{0}^{2}\right)=0
$$

Where $L_{2}(c)-w_{0}^{2}=0 \quad$ y $\quad L_{3}(c)-L_{1}(c) w_{0}^{2}=0$

that is to say, $L_{3}(c)=L_{1}(c) L_{2}(c)$

Let $c=c_{0}$ satisfy (3), and let $\lambda_{1,2}= \pm i w_{0}$ and $\lambda_{3}=\lambda_{0}$ roots of Equation (1)

$$
w_{0}=\sqrt{L_{2}\left(c_{0}\right)}
$$

In addition, it must be observed that $\left(\lambda-i w_{0}\right)\left(\lambda+i w_{0}\right)\left(\lambda-\lambda_{0}\right)=0$

$$
\lambda^{3}-\lambda_{0} \lambda^{2}+w_{0}^{2} \lambda+\left(-\lambda_{0} w_{0}^{2}\right)=0
$$

From equations (1) and (4) the imaginary roots are obtained:

$\lambda_{1,2}= \pm i \sqrt{L_{2}\left(c_{0}\right)}$ and $\lambda_{3}=\lambda_{0}$ 


\subsection{Velezmoro Diving Model}

Based on the model that allows three-dimensional (3D) objects to be displayed on a twodimensional (2D) screen; Velezmoro and Ipanaque [11], propose a model that allows four-dimensional (4D) objects to be displayed in 3D.

Required: four non-collinear vectors in space

$$
\hat{\mathscr{B}}=\left\{\left(e_{11}, e_{12}, e_{13}\right),\left(e_{21}, e_{22}, e_{23}\right),\left(e_{31}, e_{32}, e_{33}\right),\left(e_{41}, e_{42}, e_{43}\right)\right\}
$$

a dip that transforms a point $p=\left(p_{1}, p_{2}, p_{3}, p_{4}\right) \in 4 D$ into a point $q=\left(q_{1}, q_{2}, q_{3}\right) \in 3 D$. For example $\varphi(p)=\frac{1}{\sqrt{3}}\left(p_{1}+p_{2}+p_{3}+p_{4},-p_{1}+p_{2}+p_{3}-p_{4},-p_{1}-p_{2}+p_{3}+p_{4}\right)$

\section{Octave Package for Qualitative Analysis and Visualization of 4D System Phase Surfaces}

The AutonomousSystems4D package has been developed in octave 5.1.0, it allows you to visualize phase surfaces of ordinary differential equation systems, linear and nonlinear, that are immersed in the fourth dimension. The Octave ode45, lsode libraries have been used to solve differential equations in numerical form. It also allows calculating the Lyapunov exponents and convergence or divergence of trajectories of the system's solutions. In this case it has been adapted to work with the Lorenz system in 4D.

\subsection{Program for Graphing Phase Surfaces of 4D Systems}

Subprogram 1 (System of Lorenz equations in 4D).

$x=0$;

function $\mathrm{xdot}=$ func $(\mathrm{x}, \mathrm{t})$

$\%$ value of the parameters of the hypercotic system.

$a=10 ; b=8 / 3 ; c=35 ; d=0.5$;

$x \operatorname{dot}(1,1)=a *(x(2)-x(1)) ; x \operatorname{dot}(2,1)=c * x(1)-x(1) * x(3)-x(2)+x(4)$;

$x \operatorname{dot}(3,1)=x(1) * x(2)-b * x(3) ; x \operatorname{dot}(4,1)=-d * x(1)$;

end

$x 0=[0,2,0,0] ; \%$ Initial conditionl

$t=$ linspace $(0,200,15000) ; \%$ time interval;

tic;

$x=l$ sode $\left({ }^{\prime}\right.$ func $\left.{ }^{\prime}, x 0, t\right) ; \%$ solves the system of $4 \mathrm{D}$ toc equations

toc;

$z=[x(:, 1) x(:, 2) x(:, 3) x(:, 4)] ; \%$ solution matrix

$\%$ Velezmoro model

$p 1=(1 / \operatorname{sqrt}(3)) \cdot *[z(:, 1)+z(:, 2)+z(:, 3)+z(:, 4)] ; p 2=(1 / \operatorname{sqrt}(3)) \cdot *[-z(:, 1)+z(:$

$, 2)+z(:, 3)-z(:, 4)]$;

$p 3=(1 / \operatorname{sqrt}(3)) \cdot *[-z(:, 1)-z(:, 2)+z(:, 3)+z(:, 4)]$;

plot3(p1,p2,p3,'b','linewidth',1.5); xlabel('X'); ylabel('Y'); zlabel('Z') 


\subsection{Program to Calculate Lyapunov's Exponents}

The method for calculating Lyapunov's exponents can be found in [4]

Subprogram 2 (Lyapunov's Algorithm).

function $[$ Texp,Lexp $]=$ lyapunov $\left(n, r h s_{-}\right.$ext_fcn, fcn_integrator, $t$ start, stept, tend,$y$ start $)$;

$\% n:$ number of non-linear OEDs. \%n2 : total number of OEDs

$\mathrm{n} 1=\mathrm{n} ; \mathrm{n} 2=\mathrm{n} 1 *(\mathrm{n} 1+1) ; \%$ Number of iterations

nit $=$ round $(($ tend-tstart $) /$ stept $) ; \%$ Memory allocation

$\mathrm{y}=\mathrm{zeros}(\mathrm{n} 2,1) ; \operatorname{cum}=$; $\mathrm{zeros}(\mathrm{n} 1,1) ; \mathrm{y} 0=\mathrm{y} ; \mathrm{gsc}=\mathrm{cum}$; znorm = cum;

$\mathrm{y}(1: \mathrm{n})=\mathrm{ystart}(:) ; \%$ Initial values

for $\mathrm{i}=1: \mathrm{n} 1 \mathrm{y}((\mathrm{n} 1+1) * \mathrm{i})=1.0$; end;

$\mathrm{t}=\mathrm{tstart}$

$\%$ Main Loop

for ITERLYAP $=1$ : nit

$\%$ Extended ODE System Solution

$[T, Y]=f e v a l\left(f c n_{-}\right.$integrator, $r h s_{-}$ext $-f c n,[t, t+$ stept $\left.], y\right)$;

$\mathrm{t}=\mathrm{t}+$ stept; $\mathrm{y}=\mathrm{Y}(\operatorname{size}(\mathrm{Y}, 1),:)$;

for $\mathrm{i}=1: \mathrm{n} 1$

for $\mathrm{j}=1: \mathrm{n} 1 \mathrm{y} 0(\mathrm{n} 1 * \mathrm{i}+\mathrm{j})=\mathrm{y}(\mathrm{n} 1 * \mathrm{j}+\mathrm{i})$; end;

end;

$\%$ Construction of a new orthonormal base using gram-schmidt

$\operatorname{znorm}(1)=0.0$;

for $\mathrm{j}=1: \mathrm{n} 1 \operatorname{znorm}(1)=\operatorname{znorm}(1)+\mathrm{y} 0(\mathrm{n} 1 * \mathrm{j}+1)^{\wedge} 2$; end;

$\operatorname{znorm}(1)=\operatorname{sqrt}(\operatorname{znorm}(1))$;

for $\mathrm{j}=1: \mathrm{n} 1 \mathrm{y} 0(\mathrm{n} 1 * \mathrm{j}+1)=\mathrm{y} 0(\mathrm{n} 1 * \mathrm{j}+1) / \mathrm{znorm}(1)$; end;

for $\mathrm{j}=2: \mathrm{n} 1$

for $\mathrm{k}=1:(\mathrm{j}-1)$

$\operatorname{gsc}(\mathrm{k})=0.0$;

for $1=1: n 1 \operatorname{gsc}(k)=\operatorname{gsc}(k)+y 0(n 1 * 1+\mathrm{j}) * \mathrm{y} 0(\mathrm{n} 1 * 1+\mathrm{k})$; end;

end;

for $\mathrm{k}=1: \mathrm{n} 1$

for $1=1:(j-1)$

$y 0(n 1 * k+j)=y 0(n 1 * k+j)-g s c(1) * y 0(n 1 * k+1) ;$

end;

end;

$\operatorname{znorm}(\mathrm{j})=0.0$;

for $\mathrm{k}=1: \mathrm{n} 1 \operatorname{znorm}(\mathrm{j})=\operatorname{znorm}(\mathrm{j})+\mathrm{y} 0(\mathrm{n} 1 * \mathrm{k}+\mathrm{j})^{\wedge} 2$; end;

$\operatorname{znorm}(\mathrm{j})=\operatorname{sqrt}(\operatorname{znorm}(\mathrm{j}))$;

for $\mathrm{k}=1: \mathrm{n} 1 \mathrm{y} 0(\mathrm{n} 1 * \mathrm{k}+\mathrm{j})=\mathrm{y} 0(\mathrm{n} 1 * \mathrm{k}+\mathrm{j}) / \mathrm{znorm}(\mathrm{j})$; end

end;

for $\mathrm{k}=1 \mathrm{n} 1 \operatorname{cum}(\mathrm{k})=\operatorname{cum}(\mathrm{k})+\log (\mathrm{znorm}(\mathrm{k}))$; end;

$\%$ normalize exponents

for $\mathrm{k}=1: \mathrm{n} 1$

$\operatorname{lp}(\mathrm{k})=\operatorname{cum}(\mathrm{k}) /(\mathrm{t}-\mathrm{tstart})$

end;

$\%$ Output data

if ITERLYAP $==1$ 
else

$$
\mathrm{Lexp}=1 \mathrm{p} ; \operatorname{Texp}=\mathrm{t} ;
$$

$\operatorname{Lexp}=[\mathrm{Lexp} ; \mathrm{lp}] ; \operatorname{Texp}=[\mathrm{Texp} ; \mathrm{t}]$

end;

for $\mathrm{i}=1: \mathrm{n} 1$

$$
\begin{aligned}
& \text { for } j=1: n 1 \\
& \qquad y(n 1 * j+i)=y 0(n 1 * i+j) ;
\end{aligned}
$$

end;

end;

end;

end;

Subprogram 3 (Lorenz 4D system).

function $\mathrm{f}=$ exp_lyapunov4 $(\mathrm{t}, \mathrm{X})$

$$
\begin{aligned}
& \mathrm{a}=10 ; \mathrm{b}=8 / 3 ; \mathrm{c}=35 ; \mathrm{d}=0.5 ; \mathrm{x}=\mathrm{X}(1) ; \mathrm{y}=\mathrm{X}(2) ; \mathrm{z}=\mathrm{X}(3) ; \mathrm{w}=\mathrm{X}(4) ; \\
& \mathrm{Y}=[\mathrm{X}(5), \mathrm{X}(9), \mathrm{X}(13), \mathrm{X}(17) ; \mathrm{X}(6), \mathrm{X}(10), \mathrm{X}(14), \mathrm{X}(18) ; \ldots \\
& \mathrm{X}(7), \mathrm{X}(11), \mathrm{X}(15), \mathrm{X}(19) ; \mathrm{X}(8), \mathrm{X}(12), \mathrm{X}(16), \mathrm{X}(20)] ; \\
& \mathrm{f}=\mathrm{zeros}(16,1) ; \\
& \mathrm{f}(1)=\mathrm{a}^{*}(\mathrm{y}-\mathrm{x}) ; \mathrm{f}(2)=\mathrm{c}^{*} \mathrm{x}-\mathrm{y}-\mathrm{X}^{*} \mathrm{z}+\mathrm{w} ; \mathrm{f}(3)=\mathrm{x}^{*} \mathrm{y}-\mathrm{b}^{*} \mathrm{z} ; \mathrm{f}(4)=-\mathrm{d}^{*} \mathrm{x} ; \\
& \text { Jac=[-a a } 00 ; \mathrm{c}-\mathrm{z}-1-\mathrm{x} 1 ; \mathrm{y} \text { x -b } 0 ;-\mathrm{d} 000] ; \mathrm{f}(5: 20)=\mathrm{Jac}^{*} \mathrm{Y} ;
\end{aligned}
$$

end

Subprogram 4 (Calculation of Lyapunov's exponents).

$[T, R e s]=$ lyapunov(4,@exp_lyapunov4D,@ ode45,0,0.5,200,[0 20 0 0$])$;

hold on

plot(T,Res(:,1),'r', 'linewidth',2); plot(T,Res(:,2),'b','linewidth',2);

plot(T,Res(:,3),'k','linewidth',2); plot(T,Res(:,4),'g','linewidth',2);

xlabel('Time'); ylabel('Lyapunov exponents'); Res(length(Res),:);

\subsection{Program for Displaying Sensitivity under Initial Conditions}

\section{Subprogram 5}

$\%$ calculates the solutions

$\mathrm{x}=0$;

function $\mathrm{xdot}=$ func $(\mathrm{x}, \mathrm{t})$

$$
\begin{aligned}
& a=10 ; b=8 / 3 ; c=35 ; d=0.5 \\
& x \operatorname{dot}(1,1)=a *(x(2)-x(1)) ; x \operatorname{dot}(2,1)=c * x(1)-x(1) * x(3)-x(2)+x(4) ; \\
& x \operatorname{dot}(3,1)=x(1) * x(2)-b * x(3) ; x \operatorname{dot}(4,1)=-d * x(1) ;
\end{aligned}
$$

end

$x 0=[0,2,0,0] ; \mathrm{X} 0=[0,2,0,0.1] ; t=\operatorname{linspace}(0,60,200) ;$ tic $;$

$\mathrm{x}=$ lsode('func', $\mathrm{x} 0, \mathrm{t}$ );

$\mathrm{z}=\left[\mathrm{t}^{\prime}, \mathrm{x}(:, 1), \mathrm{x}(:, 2), \mathrm{x}(:, 3), \mathrm{x}(:, 4)\right] ;$ toc; $\operatorname{plot}\left(\mathrm{z}(:, 1), \mathrm{z}(:, 5),{ }^{\prime}\right.$ ', 'linewidth',1.5);

xlabel('T','FontSize',14); ylabel('w(t),W(t)','FontSize',14); hold on; tic; $\mathrm{X}=1$ sode('func', $\mathrm{X} 0, \mathrm{t}) ; \mathrm{Z}=[\mathrm{t}$ ', $\mathrm{X}(:, 1), \mathrm{X}(:, 2), \mathrm{X}(:, 3), \mathrm{X}(:, 4)]$; toc;

$\operatorname{plot}\left(\mathrm{Z}(:, 1), \mathrm{Z}(:, 5), \mathrm{b}^{\prime}\right.$, 'linewidth',1.5) 


\section{Qualitative Analysis of the Lorenz Hyperchaotic System in $R^{4}$}

In this section, the AutonomousSystems4D package (see Sectión 3) is applied to perform the qualitative analysis of the Lorenz System (5), as well as to visualize the phase surfaces that are in 4D.

In ( [6]), the four-dimensional (4D) Lorenz System was proposed, with the equations of state:

$$
x^{\prime}=a(y-x) \quad y^{\prime}=c x-y-x z+w \quad z^{\prime}=x y-b z \quad w^{\prime}=-d x
$$

Where $x, y, z$ and $w$ are state variables; $a, b, c$ y $d$ are positive control parameters of the new Lorenz 4D system. This system is hypercotic for $\mathrm{a}=10, \mathrm{~b}=8 / 3, \mathrm{c}=38$ and $\mathrm{d}=$ $0.5[6]$.

\subsection{Sensitivity in Initial Conditions}

To analyze the sensitivity in the initial conditions, perturbations are made in the initial condition of each variable. For $x=0$ and $x=0.1$. See Figure $1(\mathrm{left}) ; \mathrm{y}=2, \mathrm{y}=2.1$. See Figure 1 (right); similarly for $\mathrm{z}=0$ and $\mathrm{z}=0.1 ; \mathrm{w}=0, \mathrm{w}=0.1$. It can be seen that the paths diverge in time.
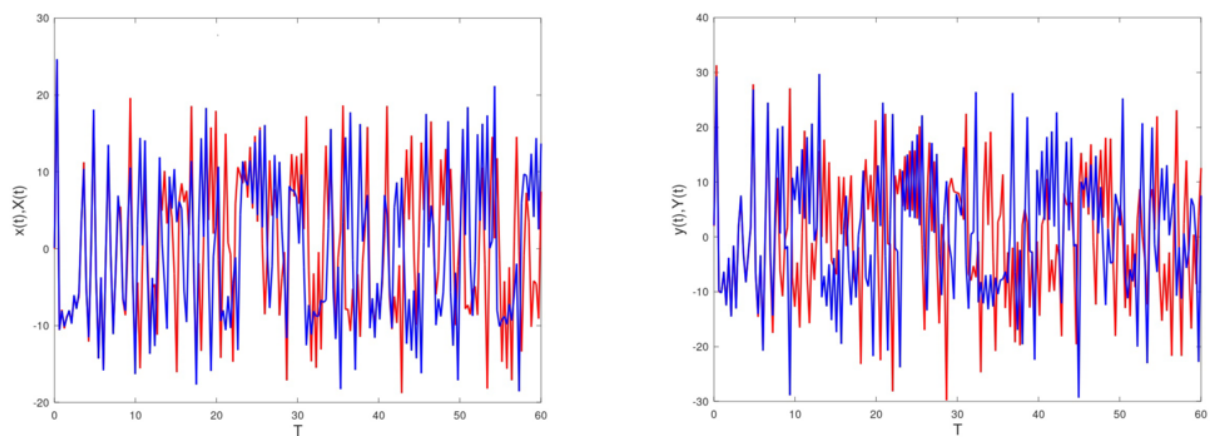

Figure 1. Disturbance to initial condition $(0,2,0,0)$

\subsection{Lyapunov's Exponents}

If in (5), the value of the control parameters are: $a=10, b=8 / 3, c=35, d=0.5$ and initial condition $(\mathrm{x}, \mathrm{y}, \mathrm{z}, \mathrm{w})=(0,2,0,0)$; the Lorenz $4 \mathrm{D}$ System has three positive exponents and one negative Lyapunov exponent (See Figure 2 left)

$$
\begin{aligned}
& \lambda_{1}=0.996745, \lambda_{2}=0.011837, \lambda_{3}=0.010623, \lambda_{4}=-14.681099 \\
& \text { Suma }=\lambda_{1}+\lambda_{2}+\lambda_{3}+\lambda_{4}=-13.662<0
\end{aligned}
$$

The Lyapunov dimension of the hyper-caotic attractor is fractional for parameter values, $a=10, b=8 / 3, c=35, d=0.5$ and initial conditions $(x, y, z, w)=(0,2,0,0)$.

$D_{L Y}=j+\frac{1}{\left|\lambda_{j+1}\right|} \sum_{i=1}^{j} \lambda_{i}=3+\frac{1}{|-14.681099|}(0.996745+0.011837+0.010623)=3.0694$ 

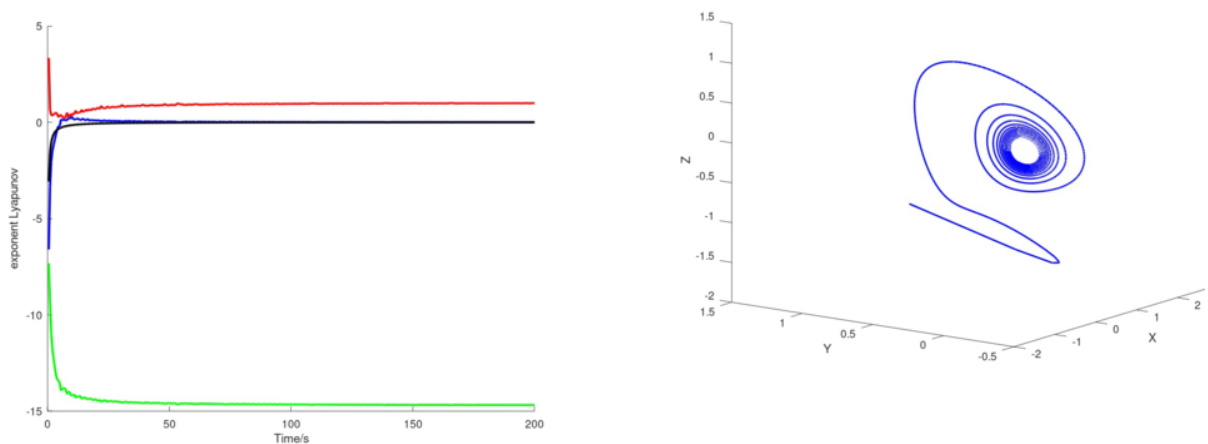

Figure 2. Lyapunov exponents (left), Hopf bifurcation (right)

\subsection{Dissipation and Existence of the Attractor}

The system divergence (5) is defined by: $\nabla V=\frac{\partial x^{\prime}}{\partial x}+\frac{\partial y^{\prime}}{\partial y}+\frac{\partial z^{\prime}}{\partial z}+\frac{\partial w^{\prime}}{\partial w}$

And it measures how quickly volumes change under system flow.

$$
\nabla V=-a-1-b=-\frac{41}{3} ; V(t)=V(0) e^{-\frac{41}{3} t}
$$

When $t \rightarrow \infty$ the volume is reduced exponentially to zero. Any solution that enters the attractor will remain in time.

\subsection{Vector Field Analysis}

Applying the coordinate transformation to the original system: $(x, y, z, w) \rightarrow(-x,-y, z,-w)$, if the equations of state remain unchanged, it means that the phase surface of the system is symmetrical with respect to the z-axis.

$$
\begin{aligned}
& x^{\prime}=-a((-y)-(-x))=-x^{\prime} \\
& y^{\prime}=-c(-x+(-y)+(-x) z-(-w))=-y^{\prime} \\
& z^{\prime}=(-x)(-y)-b z=z^{\prime} \\
& w^{\prime}=d(-x)=-w^{\prime}
\end{aligned}
$$

There is a symmetry with respect to the $z$ axes in the vector field. What happens when the flow of the system of equations crosses the coordinate axes, in the $z$ axes, $x^{\prime}=y^{\prime}=0$ and $z^{\prime}=-b z$. This indicates that every orbit that passes through the $z$ axes cannot leave it and therefore is invariant.

\subsection{Calculation of the Equilibrium Point}

To find the equilibrium point, each Lorenz 4D system equation is equated to zero.

$$
a(y-x)=0 \quad c x-y-x z+w=0 \quad x y-b z=0 \quad-d x=0
$$

The equilibrium point obtained is $P_{0}=(0,0,0,0)$ 


\subsection{Linearization of the Lorenz 4D Hyperchaotic System}

Jacobian matrix of the Lorenz system of equations 4D:

$$
J=\left(\begin{array}{cccc}
-a & a & 0 & 0 \\
c-z & -1 & -x & 1 \\
y & x & -b & 0 \\
-d & 0 & 0 & 0
\end{array}\right)
$$

Jacobian matrix of the system evaluated at break-even $(0,0,0,0)$ :

$$
A=\left(\begin{array}{cccc}
-a & a & 0 & 0 \\
c & -1 & 0 & 1 \\
0 & 0 & -b & 0 \\
-d & 0 & 0 & 0
\end{array}\right)
$$

\subsection{Calculation of Eigenvalues}

$$
\begin{gathered}
\operatorname{det}(A-\lambda I)=(\lambda+b)\left(\lambda^{3}+(a+1) \lambda^{2}+(a-a c) \lambda+a d\right)=0 \\
\operatorname{Be} L_{1}=a+1, L_{2}=a(1-c), L_{3}=a d
\end{gathered}
$$

Cubic polynomial discriminant for values of parameters $a=10, b=8 / 3 \mathrm{y} \mathrm{d}=0.5$.

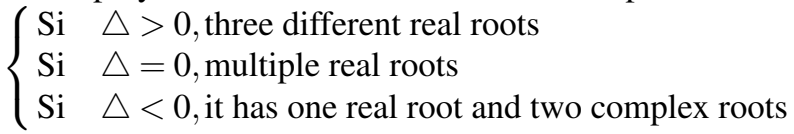

\subsection{Stability Analysis at Origin}

With the parameters $\mathrm{a}=10, \mathrm{~b}=8 / 3, \mathrm{~d}=0.5$ and $\mathrm{c}$ variation parameter.
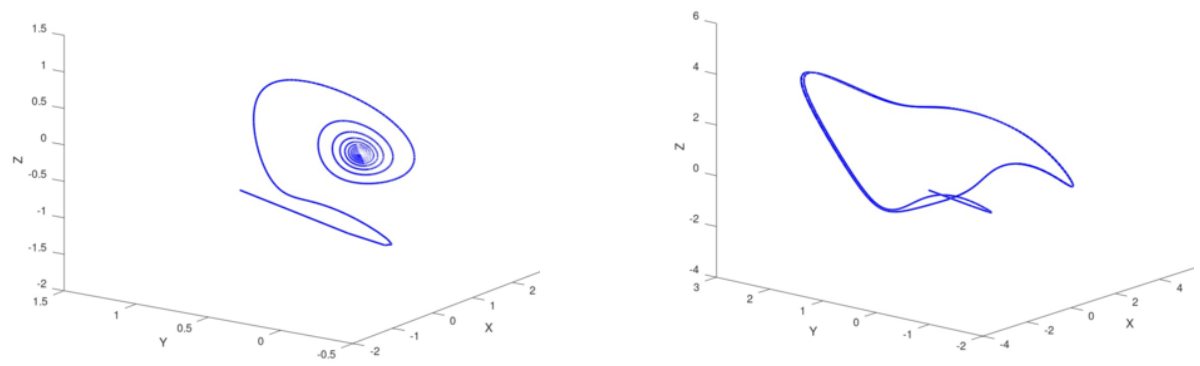

Figure 3. For $\mathrm{c}=0.9$ (left) the orbits tend to the origin, at $\mathrm{c}=2.5$ (right), the orbits have a stable limit cycle.

Case 1: When $0<c<0.95455$, the origin is asymptotically stable, there are two negative real eigenvalues and two imaginary eigenvalues that have a negative real part, therefore, all paths converge to the equilibrium point $(0,0,0,0)$ (see Figure 3 , left).

Case 2: When $0.95455<c<2.53$, the equilibrium point $(0,0,0,0)$ is unstable, there are two negative real eigenvalues and two imaginary eigenvalues that have a positive real part, in this interval a limit cycle occurs (See Figure 3, right). 
Case 3: When $c \geq 2.53$, the equilibrium point $(0,0,0,0)$ is saddle, there are two positive and two negative eigenvalues (See Figure 4). In this interval the origin becomes unstable, the trajectories move away and tend to limit attractor cycles.
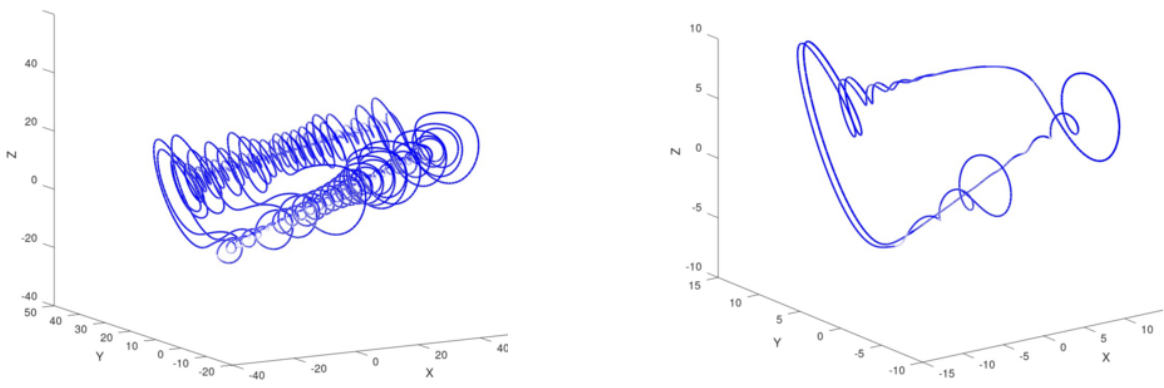

Figure 4. For $\mathrm{c}=20$ (left) and $\mathrm{c}=7$ (right), the orbits tend to two limit cycles of attraction.

\subsection{Hopf Bifurcation in the Origin.}

$$
L_{3}=L_{1} * L_{2} ; a d=(a-a c)(a+1) \Rightarrow c=\frac{a-d+1}{a+1}=c^{*}
$$

Hopf bifurcation occurs when $\mathrm{c}=0.95455$ and the eigenvalues are $\lambda_{1}=-8 / 3, \lambda_{2}=$ $0.67420 \mathrm{i}, \lambda_{3}=-0.67420 \mathrm{i}, \lambda_{4}=-11$ (See Figure 2).

\subsection{Scenarios Presented by the Lorenz 4D System by Varying the Parameter $c$}

The system (5) muestra diferentes hiper-atractores extraños de Lorenz, shows different strange Lorenz hypertractors, with fixed parameters $a=10, b=8 / 3, d=0.5$ and the parameter c variable. For $\mathrm{c}=21,22,35,40$ the origin is unstable, all the orbits move away from the origin and tend to two limiting attractor cycles (See Figures 5 and 6).
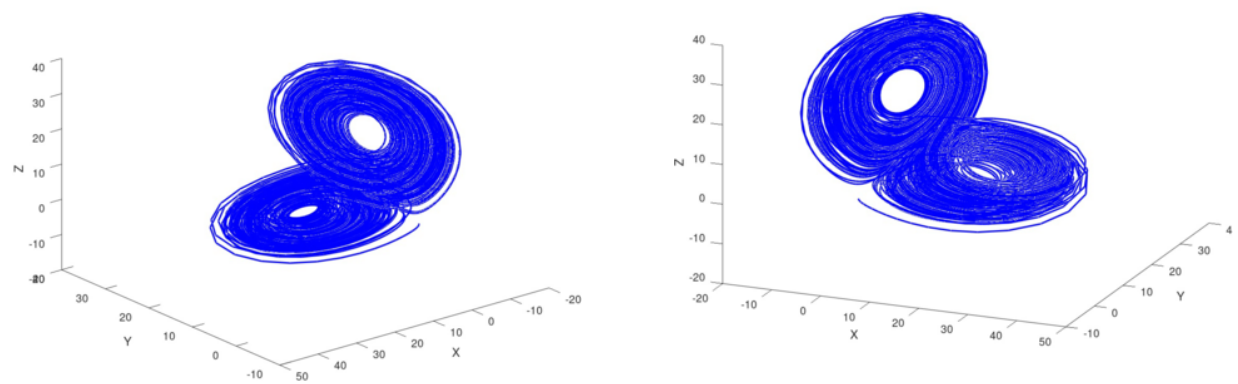

Figure 5. Display of the Lorenz Hyperattractor 4D: in $c=21$ (left), in $c=22$ (right) 

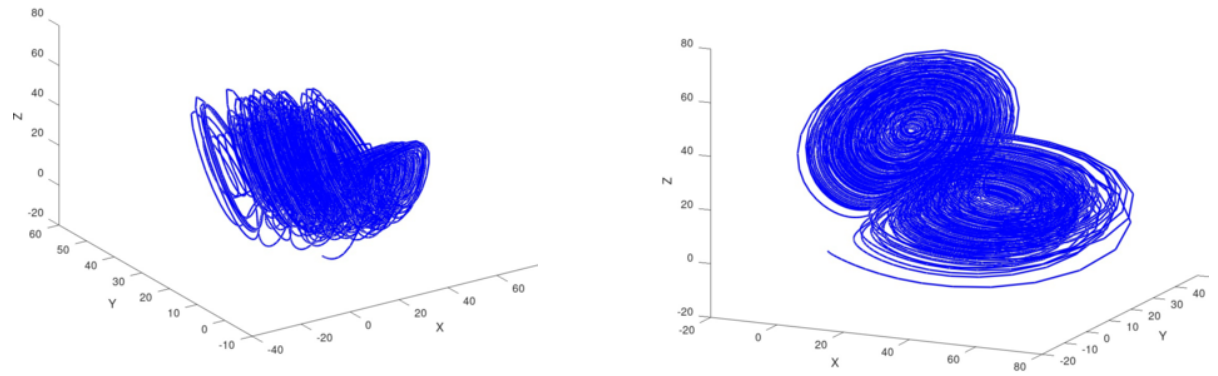

Figure 6. Display of the Lorenz Hyperattractor 4D: in $\mathrm{c}=35$ (left), in $\mathrm{c}=40$ (right)

\section{Conclusions}

The AutonomousSystems4D, package allows qualitative analysis of ordinary non-linear differential equation systems in four dimensions, as well as visualization of phase surfaces. As a case study applied to the new Lorenz 4D System, sensitivity was found in the initial conditions, Lyapunov exponents, Kaplan Yorke dimension, a stable and unstable critical point, limit cycle, Hopf bifurcation, and hyperattractors.

\section{References}

[1] Yu F, Gao L, Gu K, Yin B, Wan Q, \& Zhou Z. A fully qualified four-wing four-dimensional autonomous chaotic system and its synchronization. Optik. 2017; 131:79-88.

[2] Vaidyanathan S, Tlelo-Cuautle E, Muñoz-Pacheco J, \& Sambas A. A new four-dimensional chaotic system with hidden attractor and its circuit design. In 2018 IEEE 9th Latin American Symposium on Circuits \& Systems (LASCAS). 2018; 1-4.

[3] Benkouider K, Bouden T, \& Halimi M. Analysis, circuit implementation and active control synchronization of a new 4D chaotic system with two quadratic nonlinearities. In 2019 EEE 4th world conference on complex systems (WCCS). 2019; 1-6.

[4] Kuznetsov YA. Elements of applied bifurcation theory. Applied mathematical sciences.1998; (112): 591.

[5] Verhulst F. Nonlinear Differential Equations and Dynamical Systems. Springer. 1996.

[6] Gang-Quan S, Hui C, Yan-Bin Z. A new four-dimensional hyperchaotic Lorenz system and its adaptive control. Chinese Physics B. 2012; 20(1): 010509.

[7] Ma J, Yang Y. Hyperchaos numerical simulation and control in a 4D hyperchaotic system. Discrete Dynamics in Nature and Society. 2013; 980578.

[8] Li C,Sprott JC. Coexisting hidden attractors in a 4-D simplified Lorenz system. International Journal of Bifurcation and Chaos.2014;24(03):1450034.

[9] Panahi S, Pham V, Rajagopal K, Boubaker O, \& Jafari S. A new four-dimensional chaotic system with no equilibrium point. In Recent advances in chaotic systems and synchronization. Academic Press. 2019; 63-76.

[10] Tamba V, Kengne R, Kingni S, \& Fotsin H. A Four-Dimensional Chaotic System With One or Without Equilibrium Points: Dynamical Analysis and Its Application to Text Encryption. In Recent Advances in Chaotic Systems and Synchronization. Academic Press. 2019; 277-300.

[11] Velezmoro R, Ipanaqué R, \& Mechato JA. A Mathematica Package for Visualizing Objects Inmersed in $R^{4}$. In International Conference on Computational Science and Its Applications (ICCSA). Springer, Cham. 2019; 479-493. 